\title{
Gödel's universe
}

\section{The legacy of one of the greatest thinkers of the twentieth century.}

\section{Incompleteness: The Proof and}

Paradox of Kurt Gödel

by Rebecca Goldstein

W. W. Norton: 2005.288 pp. $\$ 22.95$.

To be published in the UK by Weidenfeld of Nicolson.

A World Without Time: The
Forgotten Legacy of Gödel
and Elnstein
by Palle Yourgrau
Basic Books/Allen Lane: 2005.224 pp. $\$ 24 / £ 20$
Martin Davis

When the US magazine Time announced its selection of the "twenty greatest thinkers and scientists of the twentieth century", readers would hardly have been surprised to find that Albert Einstein was included. But how many readers, seeing the name Kurt Gödel on the list, would have had any idea who he was, or what he had done to deserve this accolade? Both these well-written books tell the story of the life of this strange and tormented man, and explain some of his accomplishments.

Born in 1906 to a German-speaking family in Brno (which is today in the Czech Republic), Gödel was educated at university in Vienna, at first studying physics but soon finding that mathematics was his true métier. He was particularly attracted by the rigour of mathematical methods and the certainty of mathematical truth, so the controversies over the validity of these methods that raged during the 1920 s fascinated him.

A great battle for the soul of mathematics was being fought between David Hilbert, arguably the greatest mathematician of his time, and the dissident upstart L.E J.Brouwer, who vehemently denied the validity of some of the most cherished methods in the mathematician's armoury. Hilbert fumed that Brouwer and his allies "seek to provide a foundation for mathematics by pitching overboard whatever discomforts them".

Hilbert's radical remedy was to create an entirely new branch of mathematics, dubbed metamathematics, which would apply mathematical methods to mathematics itself. In order to achieve this, it was first necessary to present mathematics as a 'formula game' in which the propositions of mathematics were represented as mere assemblages of symbols, and the methods of inference that led from axioms to theorems were presented as transparent rules for manipulating symbols.

Previous work by Gottlob Frege and by the mighty team of Alfred North Whitehead and Bertrand Russell had shown how to develop all of mathematics in this manner.

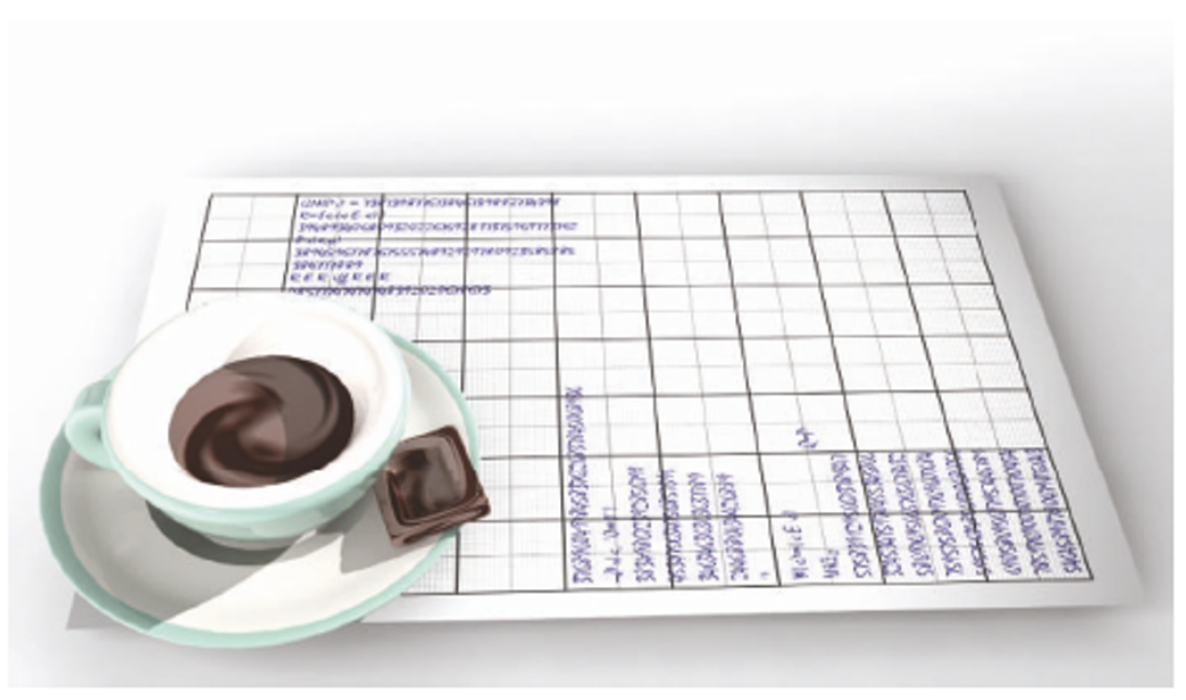

Hilbert and his co-workers were trying to prove that no contradiction would ever arise in the formula game, restricting themselves to methods even more severe than those countenanced by Brouwer.

The problem that Gödel chose for his doctoral dissertation was posed in a little textbook on formal logic published in 1928 by Hilbert and one of his students: to prove that the methods of inference used in the formula game encompass all possible logical reasoning.

After a quick success, Gödel chose for his Habilitation (the second doctorate required in the German-speaking world for an academic career) a central problem of Hilbert's metamathematics: the consistency of the arithmetic of real numbers. Hilbert's followers were already making good progress, or so it seemed, on the easier problem of proving the consistency of the arithmetic of whole numbers. So Gödel proposed to prove the consistency of the arithmetic of real numbers relative to that of whole numbers; that is, to show that any inconsistency with real numbers would in turn produce an inconsistency with whole numbers.

It was while Gödel was working on this problem that he came to an amazing conclusion that transformed him from a supporter of Hilbert's metamathematics into its nemesis. He saw that no version of the formula game could encompass all truths, even about whole numbers. In the published version of his discovery, Gödel introduced a code by which the expressions of the formula game were each represented by a single whole number. Thus, certain statements about numbers could be seen by someone privy to the code as making assertions about the formula game as well. Gödel showed how to construct a statement $P$ that, in this manner, asserted that a certain other statement, $Q$, could not be proved using the rules. Then he showed how to make $Q=P$, so that the statement $\mathrm{P}$ actually asserted that $\mathrm{P}$ itself is unprovable. From this it follows that $\mathrm{P}$ must be true and therefore, because of what it asserts, unprovable.

This necessary incompleteness of the formula game was the first blow to Hilbert's work. A few weeks later, Gödel delivered the knockout punch by proving that Hilbert's cherished goal of proving the consistency of the formula game through restricted methods was doomed to failure.

Rebecca Goldstein is a distinguished author of several novels that reflect her background as a philosopher. She writes with a although they should be warned that she is not always entirely accurate. Assuming that the particular code that Gödel used for his proof had special properties needed to make it work, she argues that "superhuman efforts" were needed to settle on that code. Actually, any other simple code would work as well. She properly emphasizes Gödel's platonist position, according to which numbers and sets have an objective existence, but she doesn't see that he only adopted this view years after his sensational proof of incompleteness, so she misses the important fact that Gödel set out not to bury Hilbert's programme but to contribute to it. Finally, she quotes Hilbert as saying: "Mathematics is a game played according to certain simple rules with meaningless marks on paper." It seems doubtful that Hilbert ever said this. For him, the formula game was not the be-all and end-all of mathematics, but only a means to an end, securing mathematics from contradiction.

Palle Yourgrau is a philosopher who has light touch that readers are sure to enjoy, 
written extensively about Gödel's contribution to Einstein's theory of relativity. Yourgrau and Goldstein both write about the friendship that developed between these two very different scholars. Gödel was a shy, reclusive man with a tendency to paranoia, whereas Einstein was worldly and outgoing. Nevertheless, the two were seen each day walking together to and from their offices at the Institute for Advanced Study in Princeton, obviously enjoying one another's company.

The equations of general relativity, having superseded Newton's account of gravitation, provide one of the great successes of twentieth-century physics. In 1949, Gödel discovered unexpected solutions to these equations corresponding to universes in which no universal temporal ordering is possible.A hypothetical inhabitant of such a universe could, in principle, travel to his own past. Yourgrau argues that because time fails to exist in these Gödel universes, and because the differences between such universes and our own are accidental, time can't exist in our world either. I doubt that many readers would be convinced by this argument.

When his wife was hospitalized, Gödel literally starved himself to death, unwilling to eat anything not prepared by her. Referring to his sad end, Goldstein makes the untoward suggestion that he might have imagined that he was living in an actual Gödel universe in which he could look forward to an eternal recurrence, reliving his life over and over again. A staunch believer in an afterlife, Gödel would hardly have sought such a fate. In any case, weird as the Gödel universes are, such eternal recurrence is not one of their properties.

Martin Davis is professor emeritus, New York University, and is a visiting scholar at the University of California, Berkeley. He can be contacted at 3360 Dwight Way, Berkeley, California 94704-2523, USA.

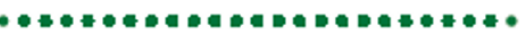 The dark side of astronomy}

Empire of the Stars: Friendship, Obsession and Betrayal in the Quest for Black Holes

by Arthur I. Miller

Little, Brown/Houghton Mifflin: 2005. 416 pp. £16.99/\$26

\section{Thanu Padmanabhan}

Science, unfortunately, is done by humans. This makes scientific debates subject to the usual follies of human interaction, prejudices and lack of objectivity. One classic example in the modern history of astrophysics is the dispute between Arthur Eddington and Subrahmanyan Chandrasekhar (also known as Chandra) regarding the fate of stars with masses above a critical value. In 1930, at the age of just 19, Chandra made the pioneering discovery that such objects should continue to collapse to a singularity, a mathematical point of infinite density. Eddington, the leading authority in astrophysics at the time, ridiculed this conclusion. And thereby hangs a tale.

The fate of such massive objects is an extremely important question in astrophysics. Chandra was predicting the existence of black holes, although this idea was years away from reaching maturity. $\mathrm{He}$ reached his viewpoint by combining the laws of special relativity with those of quantum theory as applied to particles such as electrons when they are in a state in which they are said to be relativistically degenerate. Chandra's calculations, related to the pressure of the relativistically degenerate gas, led him to the conclusion that gravity will inexorably crush a sufficiently massive body to a point.

If Eddington had merely expressed discomfort at matter collapsing to a point-like singularity of infinite density, that would have been fair enough. Even today we do not understand what actually happens to matter that falls into a black hole, and those

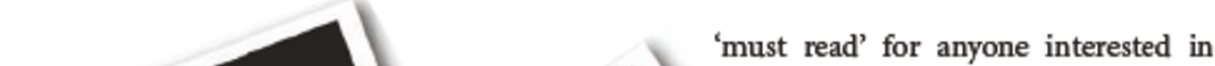
astrophysics or the history of science. The book begins with the dramatic moment when Chandra gave a seminar on his results at a meeting of the Royal Astronomical Society in London in January 1935, and describes how Eddington made mincemeat of it. It then goes backwards in time, caricaturing both of the dramatis personae, Chandra and Eddington. Having put the pieces together, Miller goes on to describe how this particular episode left its mark on Chandra. As well as the story of Chandra's encounter with Eddington, Miller tells of the progress made in this subject over the years, and an appendix of us who have thought uncomfortable with the notion of a singularity. But Eddington questioned Chandra's logic and his calculation, and raised irrelevant objections. Later work completely vindicated Chandra, but

the early humiliation left a deep scar. We can only speculate about what would have happened if Eddington had taken the scientifically correct stand and helped to develop Chandra's ideas further.

Historian Arthur Miller tells this story in a lively and exciting narrative in Empire of the Stars. The book is so beautifully written that $I$ read it in one sitting. The scientific issues, as well as the personalities of everyone involved in the debate, have been described in picturesque detail without either mincing words or making unsubstantiated claims. Wherever necessary, Miller is careful to give references to the sources from which he has gathered the information, making the work fairly authoritative. In short, this book is a 